\title{
Web Based Interactive Tutorials in Obstetrics and Gynecology
}

\author{
${ }^{1}$ Sanja Plavsic Kupesic, ${ }^{2}$ Bhargavi Patham, ${ }^{3}$ Martha Guerra \\ ${ }^{1}$ Professor and Clinical Professor of Obstetrics and Gynecology and Radiology, Department of Medical Education, Paul L \\ Foster School of Medicine, Texas Tech University Health Sciences Center, 5001 El Paso Drive, El Paso, Texas 79905, USA \\ ${ }^{2}$ Assistant Professor and Pathophysiology and Cell Biology, Department of Medical Education, Paul L Foster School of Medicine \\ Texas Tech University Health Sciences Center, El Paso, Texas 79905, USA \\ ${ }^{3}$ Associate Director for Instructional Design, Office of Curriculum, Evaluation and Accreditation, Department of Medical Education \\ Paul L Foster School of Medicine, 5001 El Paso Drive, El Paso, Texas 79905, USA
}

Correspondence: Sanja Plavsic Kupesic, Professor and Clinical Professor of Obstetrics and Gynecology and Radiology Department of Medical Education, Paul L Foster School of Medicine, Texas Tech University Health Sciences Center, 5001 El Paso Drive, El Paso, Texas 79905, USA, Phone: (915) 783-1700, Fax: (915) 783-1715, e-mail: sanja.kupesic@ttuhsc.edu

\begin{abstract}
The challenge of integrating basic science and clinical knowledge contents has become an educational imperative at Paul $L$ Foster School of Medicine clinical presentation based curriculum. During the first two years of our curriculum, students are introduced to clinical applications of basic science knowledge. For better understanding and appreciation of the important links between the basic science principles and clinical medicine, students return to basic science topics. To support such a progression of students' knowledge and skills, the curriculum should allow and support individual learning. Interactive tutorials created by authors of different backgrounds and expertise facilitate clinical application of basic science knowledge and provide multiple pathways in learning and inductive reasoning. Web based learning modules do not supplement traditional lectures, small groups sessions and team based learning activities, but they may significantly enrich students' learning experience.

Physical and content boundaries between basic science and clinical departments are sometimes difficult to breach. In virtual space, thematically organized modules and collective approach of interdisciplinary teaching teams allow full integration of core content. This concept of distance learning contributes to educational continuity, longitudinal experience and educational mobility.
\end{abstract}

Keywords: Web based interactive tutorials, basic science, clinical science, educational continuity, longitudinal experience.

\section{WEB BASED LEARNING MODULES FOR STUDENTS}

The comprehensiveness and quality of Obstetrics and Gynecology clerkship and residency program websites vary considerably. ${ }^{1,2}$ Most of them provide information on specific topics, which correspond to peer review medical journals, but do not use multidisciplinary approach and do not facilitate integration between basic science disciplines and clinical content. The concept of educational continuity is crucial for connection between basic science and clinical medicine. ${ }^{3}$ A series of clinical experiences, developmentally appropriate for students' stage of learning are necessary for better understanding of the dynamics of patients' care. ${ }^{4}$ There is no doubt that hospital environment, inpatient services, ambulatory setting and community experiences are necessary to learn clinical medicine and gain clinical experience. In many situations students and residents are not able to explore and experience the evolution of patients' symptoms, and monitor the clinical course of the disease, especially once patients resume the ambulatory based care. In these cases the use of educational technology is necessary for filling the gaps in clinical experiences for students and residents.

Thermatically organized modules enable longitudinal tracking of natural causes of the disease and complications in virtual patients. Imaging studies are an integral part of the web based tutorials which facilitate learning and understanding of human anatomy and pathology. Underlying information, from pathophysiology, microbiology, and pharmacology to family and community allow learners' longitudinal experience. At the beginning of 21 st century, use of computer based technology plays an important role in medical education and may be used for implementing innovative clinical education.

Marsh et $\mathrm{al}^{5}$ developed web based learning module on embryonic development. Their data suggest that students 
who used an interactive module performed better than those given only traditional resources if they used the module after they were already somewhat familiar with the material. These findings indicate that computer assisted instruction modules are more useful if used toward later stages of learning, rather than as initial resource. Recent study by Bryner et al ${ }^{6}$ evaluated interactive computerized modules in preclinical medical education. Their data suggest that medical students may learn more effectively and feel less intimidated by difficult concepts when interactive modules supplement traditional instruction.

Interdisciplinary teaching team of Paul L Foster School of Medicine has created a series of interactive tutorials on obstetrics and gynecology incorporating basic science disciplines (anatomy, physiology, embryology, histology, pathology, microbiology and pharmacology), clinical knowledge content, series of case reports, clinical skills portion, medical ethics topics, medical dictionary, medical image library, entry, and exit questions (Figure 1 demonstrates layout of a home page for interactive tutorial on adolescent gynecology).

Series of web based teaching modules at Paul L Foster School of Medicine consists of twelve topics describing the most common clinical presentations in the field of obstetrics and gynecology: abnormal menstrual cycle, normal pregnancy, abnormal pregnancy and pregnancy loss, infertility, contraception, menopause, pelvic floor relaxation and prolapse, screening (breast lumps, galactorrhea, Pap smear), vaginal discharge and sexually transmitted disease, abnormal genital tract bleeding, pelvic mass and pelvic pain. By learning the basic and clinical sciences synchronously, within the context of most common clinical presentations in reproductive medicine, learners are achieving a high level of integration.

The editors and authors of the web based interactive tutorials in obstetrics and gynecology anticipate the following outcomes:

1. Better integration between the basic and clinical sciences in the field of obstetrics and gynecology.

2. Improved longitudinal integration and retention of knowledge.

3. Case reports and imaging component of the web based tutorials, organized according to the clinical presentations enable students and postgraduates to use an inductive reasoning paradigm in solving clinical problems from every day routine practice.
4. Medical educators who will review the website will be able to create similar projects at their institutions in their respective specialties to better integrate basic and clinical sciences and to encourage expert clinical reasoning throughout the medical education process (from the first year of Medical School through residency and beyond).

Series of online interactive web based tutorials in obstetrics and gynecology incorporating all basic science disciplines, clinical knowledge component, series of case reports, medical skills portion, medical ethics topics, medical dictionary, medical image library, and entry and exit questions is currently located at the public site of the Texas Tech server, allowing regional, national and international dissemination of the project.

\section{WEB BASED LEARNING MODULES FOR POSTGRADUATES}

During the last 30 years, diagnostic ultrasound has become a common practice in obstetrics and gynecology, particularly for challenging cases such as identification of gynecologic malignancy, ectopic pregnancy or fetal abnormality. Health care institutions in both developed and developing countries are widely equipped with ultrasound equipment, while the knowledge and skills necessary for implementing are underdeveloped. The improvement of ultrasound diagnosis has potential to reduce maternal and infant morbidity and save lives, especially in developing countries.

With the help of the members of Ian Donald InterUniversity School of Ultrasound in Obstetrics and Gynecology, we continue to develop extensive distance teaching instruments comprised of interactive tutorials and comprehensive image bank and clinical case gallery. These tools will be used to enhance the learning and understanding of health care professionals in reproductive medicine, particularly in the field of ultrasound. In addition, web based modalities may be used to provide expert diagnosis and advice in case management in reproductive medicine.

Objectives of our scholarly educational project are the following:

1. To create a series of web based interactive tutorials in obstetrics and gynecology for the distance teaching of the following health care professionals:

a. Sonography technologists,

b. Medical students and residents, and

c. Specialists and subspecialists in obstetrics and gynecology. 


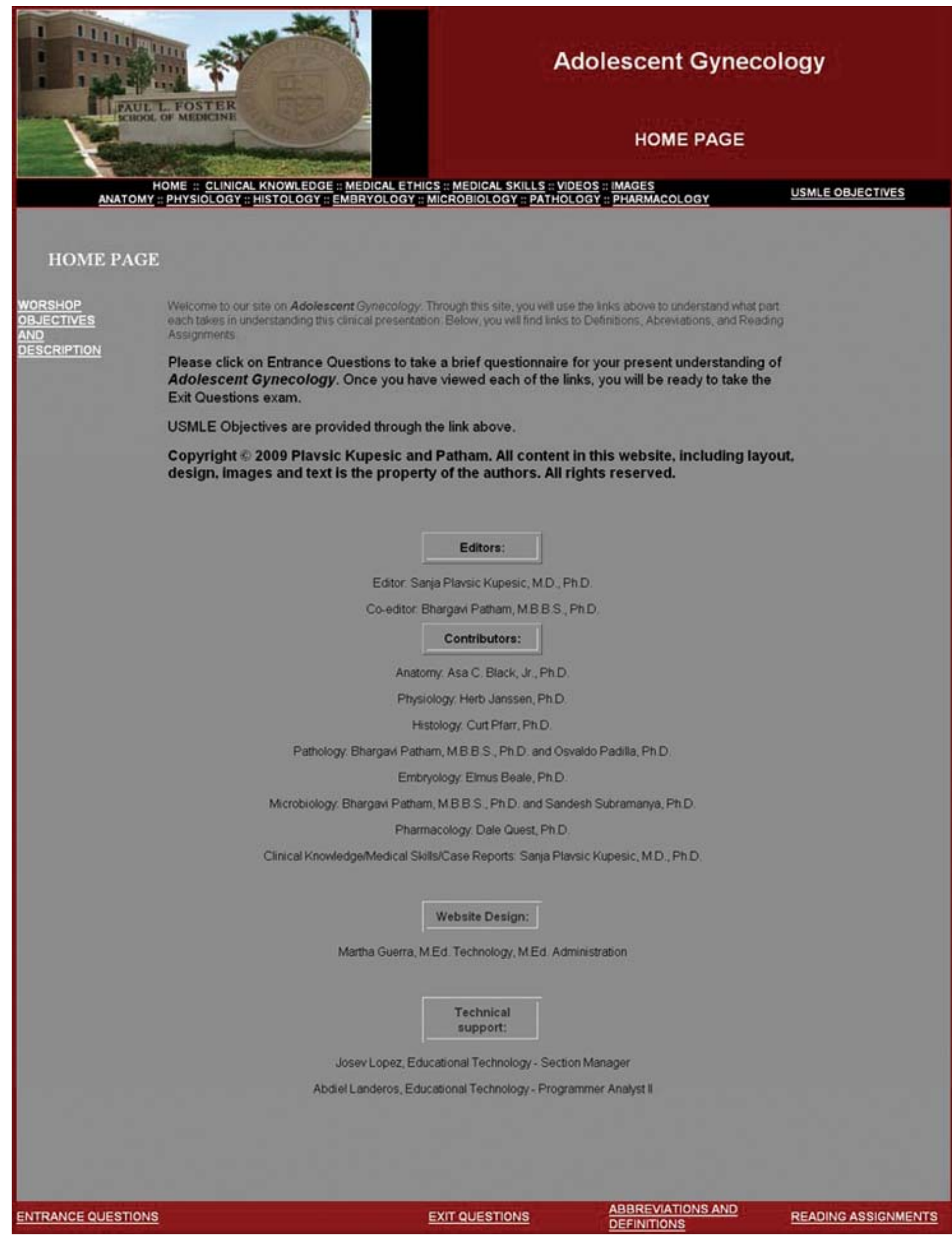

Fig. 1: Home page for online access-protected interactive tutorial on adolescent gynecology 
2. In support of the web based teaching in objective 1 , to create a comprehensive on line ultrasound image library.

3. To provide expert diagnostic and case management advice using a variety of distance teaching and web based modalities.

The goals of our project are to promote the science and art of ultrasound scanning, facilitate research and education in the field of ultrasound in reproductive and fetal medicine, and accelerate the dissemination of information.

\section{REFERENCES}

1. Foster AM, Jackson CB, Martin SB. Reproductive health and cyber (mis) representations: A content analysis of Obstetrics and Gynecology residency program websites. Contraception 2008;78(2):99-105.

2. Jain T, Barbieri RL. Website quality assessment: Mistaking apples for oranges. Fertil Steril 2005;83(3):545-47.

3. Cox M and Irby DM. "Continuity" as an organizing principle for clinical education reform. New Engl J Med 2007;356(8):85866.

4. Whitcomb ME. Ambulatory based clinical education: Flexner revisited. Acad Med 2006;81(2):105-06.

5. Marsh KR, Giffin BF, Lowrie DJ Jr. Medical student retention of embryonic development: Impact of dimension added by multimedia tutorials. Anat Sci Educ 2008;1(6):252-57.

6. Bryner BS, Sadawi-Konefka D, Gest TR. The impact of interactive, computerized educational modules on preclinical medical education. Anat Sci Educ 2008;1(6):247-51. 